\title{
VERIFICATION OF A 3-D NUMERICAL MODEL FOR SPILLWAY AERATOR
}

\author{
Mualla Ozturk \\ Firat University, Civil Engineering Department, Elazig, Turkey \\ M. Cihan Aydin \\ Bitlis Eren University, Technical Vocational School of Higher Education, Bitlis, Turkey \\ mcaydin@gmail.com,mcaydin@yyu.edu.tr
}

\begin{abstract}
In this study, the results of CFD obtained by using Fluent with respect to the air entrainment at spillway aerators are compared to the data of the physical model study and the results of some empirical equations presented by other investigators. The air entrainment rates obtained from the CFD analyses are in reasonable good agreement with the values calculated by the empirical equations. However, the CFD results are better than the physical model data including considerable scale effects. The numerical verification procedure in this study is based on the ASME editorial policy statement, which provides a framework for computational fluid dynamics uncertainty analysis. So, the validation of the CFD model was discussed in this scope.
\end{abstract}

Key Words - Air Entrainment, CFD, Fluent, Spillway Aerators

\section{INTRODUCTION}

The development of spillway aerators has been pioneered to a very large extent through the use of physical hydraulic models. However, in the studies of physical hydraulic models, most experience related with the phenomenon of air entrainment has shown that considerable scale effect can be expected. Most hydraulic structures models are designed and operated according to Froude law of similarity, with viscous and surface tension forces as represented by Reynolds and Weber numbers respectively. With air entrainment, the latter two forces are very significant and their poor model representation often results in poor scaling of model results compared to prototype experience. In laboratory conditions of an air-water flow phenomenon, Weber number similarity cannot be achieved concurrent with Froude similarity. Pinto [1] suggested that, turbulence effects in a model may be dampened due to a thicker laminar sublayer associated with a Reynolds scale effect. The laminar sublayer and surface tension forces restrain the action of turbulence and tending to reduce the effectiveness of the airentraining mechanism in the model.

Complete modeling of most hydraulic structures may become practically impossible at those scales because of high water discharges needed in the model and correspondingly due to high cost. Therefore, model studies have to take scale effects into consideration, mostly due to the viscous and surface tension phenomena. On the contrary of physical models, large hydraulic structures with the real dimensions can be simulated using one of the Computational Fluid Dynamics (CFD) methods. Recently, the numerical methods including 3-D Computational Fluid Dynamics (CFD) have been 
developed rapidly with rising computer technology and advanced numerical methods. The CFD models are more flexible and require less time, less cost and less effort than physical hydraulic models. The scale effects are also eliminated through the real dimensions of prototype used in the CFD models. The CFD analyses have widely been used involving fluid mechanics as aerodynamic, multiphase flows, free surface flows, etc.

The aim of this paper is to present a verification of the CFD models related to the air entrainment in a spillway aerator, because the using of CFD model is encouraged in the large hydraulic structures. In this respect, the CFD model which simulates the experimental model studied by Demiroz [2] was prepared and performed. The results of CFD model were compared with the experimental results including considerable scale effects and the calculated values of some empirical equations presented by other researchers. The numerical analysis results by means of FLUNET software were also verified by acceptable method based on the generalized Richardson Extrapolation and the ASME editorial policy statement.

\section{NUMERICAL MODEL}

To be able to observe the effects of model scale, the 3-D numerical model was created with the dimensions of prototype represented by Demiroz's [2] model. In the numerical model, the chute slope $(\tan \alpha)$ and the cross sectional area of the air duct were 0.30 and $1.5 \mathrm{~m}^{2}$ respectively which were constant, and the height of ramps $\left(t_{r}\right)$ were $0.10 \mathrm{~m}, 0.15 \mathrm{~m}, 0.20 \mathrm{~m}$ and $0.25 \mathrm{~m}$. The Froude numbers were considered as a range of $4.31 \leq \mathrm{Fr} \leq 7.52$. The velocities of flow in upstream of the aerator were changed from $20.27 \mathrm{~m} / \mathrm{s}$ to $26.31 \mathrm{~m} / \mathrm{s}$. The range of Reylolds numbers of water flow was approximately $15 \times 10^{6} \leq \mathrm{Re} \leq 102 \times 10^{6}$. The detail of model geometry is shown in Fig. 1 . The numerical geometry and grid construction were generated by using the Gambit software (Fig. 2). The flow was assumed as incompressible. The inlet condition of flow was fully developed, and assumed as a uniform velocity distribution.
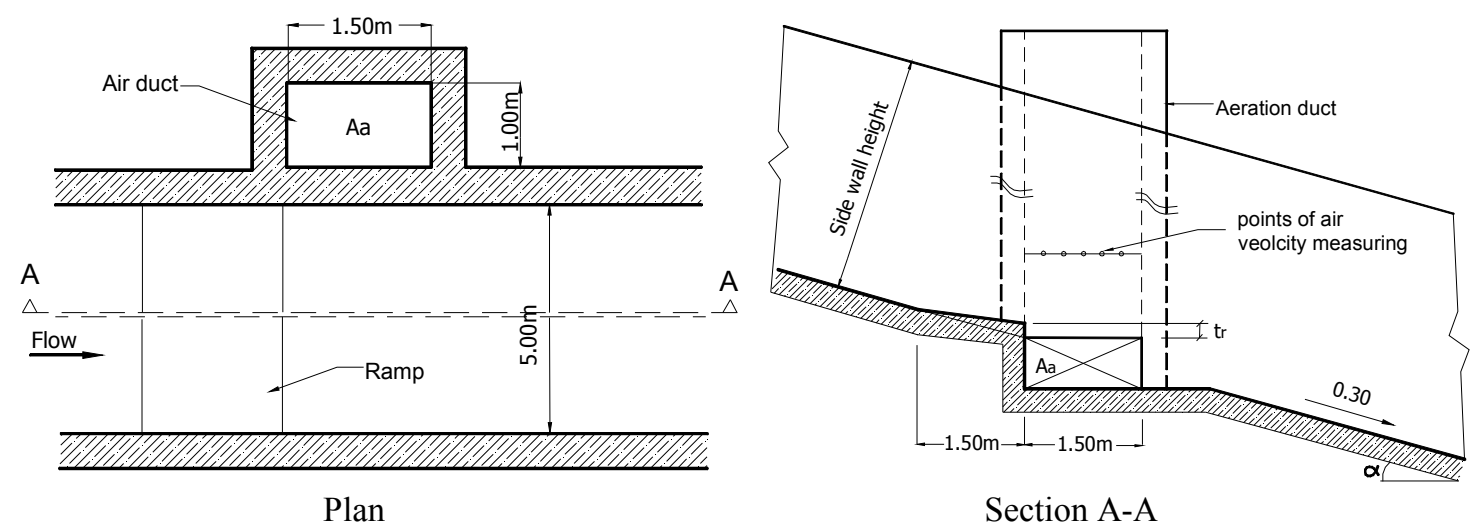

Fig. 1. The geometry of the spillway aerator with prototype dimensions.

In the numerical solutions, 3-D multiphase model (Algebraic Slip Mixture Model) and standard $k-\varepsilon$ turbulence model were used. The standard $k-\varepsilon$ model is a semiempirical model based on the model transport equations for the turbulence kinetic 
energy $(k)$ and its dissipation rate $(\varepsilon)$ [3]. The model transport equation for $k$ is derived from the exact equation, while the model transport equation for $\varepsilon$ was obtained using physical reasoning and bears little resemblance to its mathematically exact counterpart.

In the numerical solutions, the algebraic slip mixture model was preferred in FLUENT software. The algebraic slip mixture model does not assume that there is an interface between two immiscible phases; it allows the phases to be interpenetrating. Moreover, the algebraic slip mixture model allows the two phases to move at different velocities. The algebraic slip mixture model can solve the continuity equation and the momentum equation for the mixture [4].

The continuity equation for the mixture is

$\frac{\partial}{\partial \mathrm{t}}\left(\rho_{\mathrm{m}}\right)+\frac{\partial}{\partial \mathrm{x}_{\mathrm{i}}}\left(\rho_{\mathrm{m}} \mathrm{u}_{\mathrm{m}, \mathrm{i}}\right)=0$

where $\rho_{m}$ is mixture density and $\vec{u}_{m}$ is mass-averaged velocity. No mass transfer is allowed in the algebraic slip mixture model.

The momentum equation for the mixture can be obtained by summing the individual momentum equations for both phases. It can be expressed as

$$
\begin{aligned}
& \frac{\partial}{\partial t} \rho_{m} u_{m, j}+\frac{\partial}{\partial x_{i}} \rho_{m} u_{m, i} u_{m, j}= \\
& -\frac{\partial p}{\partial x_{j}}+\frac{\partial}{\partial x_{i}} \mu_{m}\left(\frac{\partial u_{m, i}}{\partial x_{j}}+\frac{\partial u_{m, j}}{\partial x_{i}}\right)+\rho_{m} g_{j}+F_{j}+\frac{\partial}{\partial x_{i}} \sum_{k=1}^{n} \alpha_{k} \rho_{k} u_{D k, i} u_{D k, j}
\end{aligned}
$$

where $n$ is number of phases; $\mu_{m}$ is viscosity of mixture; $F$ is a body force; $\alpha_{k}$ is volume fraction of phase $k$ and $\vec{u}_{D k}$ are drift velocities.

The boundary conditions that are wall, pressure inlet and velocity-inlet were defined in appropriate surfaces of the model geometry (Fig. 2). The boundaries open to atmosphere were defined as pressure inlet and relative pressure of atmosphere was taken as zero Pascal.
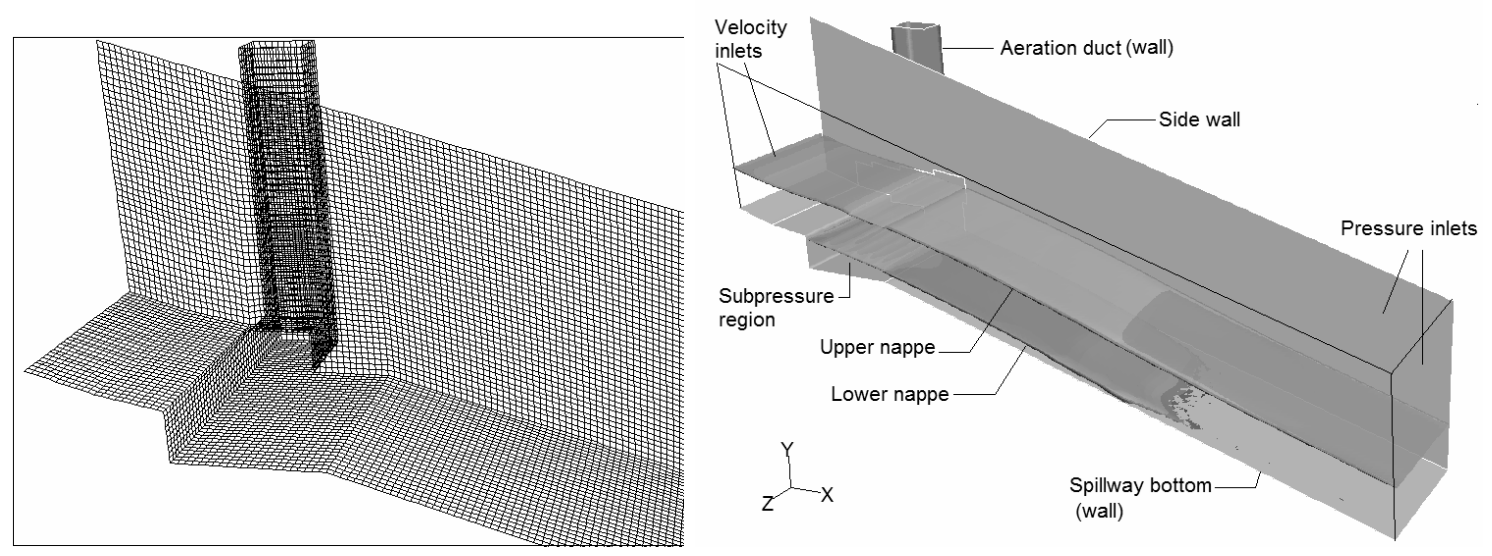

Fig. 2. The boundaries and grid construction of the 3-D numerical model 


\section{VERIFICATION OF THE CFD MODEL}

The numerical verification procedure in this study is based on the ASME editorial policy statement, which provides a framework for computational fluid dynamics uncertainty analysis [5]. Convergence investigation involves two aspects as iterative convergence and grid convergence.

\subsection{Iterative Convergence}

Before any discretization error estimation is calculated, it must be ensured that iterative convergence is achieved with at least three orders of magnitude decrease in the normalized residuals for each equation solved. For time-dependent problems, iterative convergence at every time step should be checked. Figure 3(a) shows the normalized residuals for some equations solved for only 200 of totally 8,000 iterations. Each time steps are maximum 40 iterations and all residuals drop under three orders every time steps. Figure 3(b) also shows, as a sample convergence trends, convergence history of velocity magnitude on air inlet surface which is a most important parameters in this problem. Iterative convergence is achieved in about 150 time steps (6,000 iterations) in Figure 3(b).

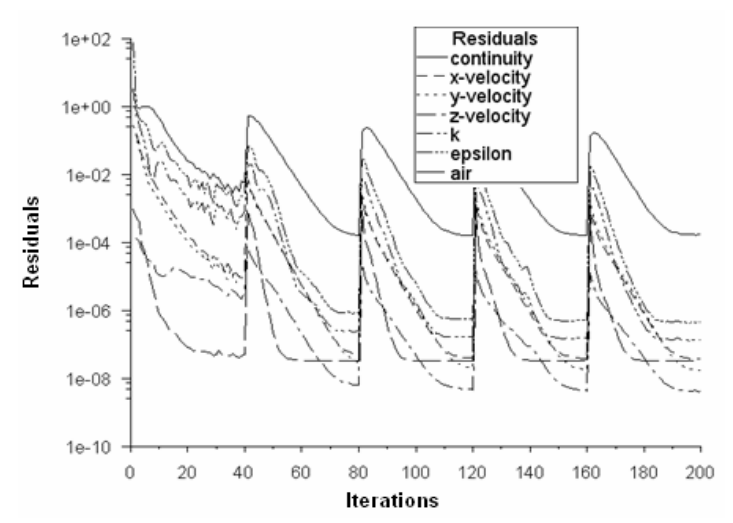

(a)

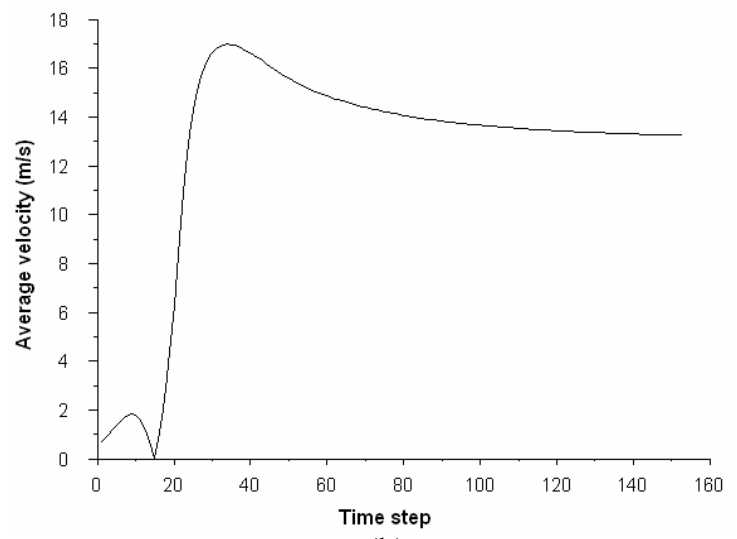

(b)

Fig. 3 (a) Time-dependent iterative convergence for $\mathrm{Fr}=7.52$,

(b) Convergence history of velocity magnitude on air inlet surface for $\mathrm{Fr}=7.52$

\subsection{Grid Convergence}

The GCI (Grid Convergence Index) method used herein is an acceptable and recommended method that has been evaluated over several hundred CFD cases. The GCI was originally proposed by Roache $[6,7,8]$ as a general method for reporting the sensitivity of model solutions to numerical discretization. This method is based on the generalized Richardson Extrapolation involving comparison of discrete solutions. Estimation of grid convergence and the associated uncertainty require a minimum of three grids. A fine-grid Richardson error estimator approximates the error in a fine-grid solution $f_{1}$, by comparing this solution to that of coarse grid $f_{2}$, and is defined as

$$
E_{1}^{\text {fine }}=\frac{\varphi}{1-r^{p}}
$$


while a coarse-grid Richardson error estimator approximates the error in a coarse-grid solution $f_{2}$, by comparing this solution to that of a fine grid $f_{l}$, and is defined as

$$
E_{2}^{\text {coarse }}=\frac{r^{p} \varphi}{1-r^{p}}
$$

where $\varphi=f_{2}-f_{1}, f_{2}$ is a coarse-grid numerical solution obtained with grid spacing $h_{2}, f_{1}$ is a fine-grid numerical solution obtained with grid spacing $h_{l}, r$ is refinement factor between the coarse and fine grid $\left(r=h_{\text {coarse }} / h_{\text {fine }}>1\right)$ and $p$ is order of accuracy. Roache [6] recommended a minimum $\% 10$ change in the grid refinement factor, $r$. For three-dimensional calculations, a representative grid size $h$ can be estimated as

$$
h=\left[\frac{1}{N} \sum_{i=1}^{N}\left(\Delta V_{i}\right)\right]^{1 / 3}
$$

where $\Delta V_{i}$ is volume of $i^{\text {th }}$ cell and $N$ is number of cell.

The GCI is defined with a safety factor for fine and coarse grids as

$$
\begin{aligned}
& G C I_{1}^{\text {fine }}=F_{s}\left|E_{1}\right| \\
& G C I_{2}^{\text {coarse }}=F_{s}\left|E_{2}\right|
\end{aligned}
$$

Ostensibly, if we have a fine-grid and a coarse-grid solution, we would be expected to use the fine-grid solutions, so reporting of the above fine-grid evaluation of GCI would be applied. It is recommended that value of $F_{s}=3$ is conservative and relates the grid convergence study to one with a grid doubling with second-order method. However, for performed grid convergence studies using three or more grid solutions, a modest value of $F_{s}=1.25$ was recommended [7].

Without an exact solution for the actual problem, it is necessary to have at least three grid solutions to extract $p$. If the grid refinement is performed with constant $r$, then the order can be extracted directly from three grid solutions.

$$
p=\ln \left(\varphi_{32} / \varphi_{21}\right) / \ln (r)
$$

But, if $r$ is not restricted to constant, the order can be calculated using the expression

$$
\begin{aligned}
& p=\frac{1}{\ln \left(r_{21}\right)}|\ln | \varphi_{32} / \varphi_{21}|+q(p)| \\
& q(p)=\ln \left(\frac{r_{21}^{p}-s}{r_{32}^{p}-s}\right)
\end{aligned}
$$

where $\varphi_{21}=f_{2}-f_{1}, \varphi_{32}=f_{3}-f_{2}, r_{21}=h_{2} / h_{1}, r_{32}=h_{3} / h_{2}$ and $s=1 \cdot \operatorname{sign}\left(\varphi_{32} / \varphi_{21}\right)$, with subscript 1 indicating finest grid in present notations. Eq. 9 can be solved using fixed-point iteration, with the initial guess equal to the first term.

The approximate relative error can be calculated as

$$
e_{21}=\left|\frac{f_{1}-f_{2}}{f_{1}}\right|
$$

The relative grid convergence index with a safety factor defined by Roache [6]: 
$G C I_{\text {fine }}=\frac{1.25 e_{21}}{r^{p}-1}$

Three significantly different set of grids are selected and then simulations are run to determine the values of key variables important to the objective of simulation study, for example variable $f$ critical to conclusions being reported that herein is the unit air discharge in the aerator duct. In Table 1, the discretization errors were indicated for three selected grids with total number of cells as 266,934, 124,720 and 52,624 as 3-D hexahedral elements. The refinement factors, not restricted as constant numbers, were estimated as $r_{21}=1.30$ and $r_{32}=1.40$ using the representative grid sizes calculated by Eq. 5 .

According to Table 1, the numerical uncertainty in the fine-grid solution is calculated as ranging from $3.3 \%$ to $12.2 \%$, which corresponds up to $\pm 0.46 \mathrm{~m}^{3} / \mathrm{s} / \mathrm{m}$ approximately.

Table 1. Discretization Errors for $t_{r}=0.15 \mathrm{~m}$ and $\tan \alpha=0.30$

\begin{tabular}{|c|c|c|c|c|c|c|c|c|c|c|}
\hline \multirow[b]{2}{*}{ Fr } & \multicolumn{3}{|c|}{$q_{a}\left(\mathrm{~m}^{3} / \mathrm{s} / \mathrm{m}\right)$} & \multirow[b]{2}{*}{$\varphi_{21}$} & \multirow[b]{2}{*}{$\varphi_{32}$} & \multirow[b]{2}{*}{$p$} & \multirow[b]{2}{*}{$e_{21}$} & \multirow[b]{2}{*}{$e_{32}$} & \multirow{2}{*}{$\begin{array}{r}\text { GCI }_{1, \text { fine }} \\
(\%)\end{array}$} & \multirow{2}{*}{$\begin{array}{r}\mathbf{G C I}_{2, \text { fine }} \\
(\%)\end{array}$} \\
\hline & $f_{1}$ & $f_{2}$ & $f_{3}$ & & & & & & & \\
\hline 7.52 & 4.22 & 4.02 & 3.88 & -0.20 & -0.14 & 2.75 & 0.047 & 0.035 & 5.6 & 2.9 \\
\hline 7.00 & 4.50 & 4.30 & 4.13 & -0.20 & -0.17 & 1.85 & 0.044 & 0.040 & 8.9 & 5.7 \\
\hline 6.73 & 4.80 & 4.60 & 4.45 & -0.20 & -0.15 & 2.43 & 0.042 & 0.033 & 5.8 & 3.2 \\
\hline 6.39 & 4.86 & 4.65 & 4.48 & -0.21 & -0.17 & 2.08 & 0.043 & 0.037 & 7.4 & 4.5 \\
\hline 5.92 & 4.92 & 4.70 & 4.52 & -0.22 & -0.18 & 2.03 & 0.045 & 0.038 & 7.9 & 4.9 \\
\hline 5.56 & 4.86 & 4.64 & 4.45 & -0.22 & -0.19 & 1.78 & 0.045 & 0.041 & 9.5 & 6.2 \\
\hline 4.98 & 4.37 & 4.29 & 4.10 & -0.08 & -0.19 & 2.03 & 0.018 & 0.044 & 3.3 & 5.6 \\
\hline 4.31 & 3.81 & 3.56 & 2.98 & -0.25 & -0.58 & 1.96 & 0.066 & 0.163 & 12.2 & 21.8 \\
\hline
\end{tabular}

\subsection{Comparison with Physical Model and Calculated Values}

The air entrainment rate for spillway aerators is defined as the ratio of air discharge induced through the lower nappe of water jet by air-supply duct to water discharge, $\beta=q_{a} / q_{w}$, where $\beta$ is the air entrainment rate, $q_{a}$ is the unit air flow discharge, and $q_{w}$ is the unit water flow discharge. In this part, the air entrainment rates obtained from CFD analysis was compared with Demiroz's physical model data. The two different calculated values of $\beta$ were also used in the comparison.

The first part of these values were calculated by Equations (13) and (14) presented by Kokpinar and Gogus [9]:

$\beta_{c 1}=0.0189\left(\frac{L_{j}}{h}\right)^{0.83}\left[\left(\frac{A_{a}}{A_{w}}\right)(1+\tan \alpha)\right]^{0.24}$

where $L_{j}$ is the jet length (m), $h$ is the flow depth (m), $A_{a}$ is the entrance area of air supply duct $\left(\mathrm{m}^{2}\right), A_{w}$ is the water flow area upstream of the aerator $\left(\mathrm{m}^{2}\right)$, and $\alpha$ is the slope angle of spillway chute. The subscript $c$ indicates a calculated value. The relative jet length, $L_{j} / h$ in Eq. 13 was calculated by equation [9] stated below: 
$\left(\frac{L_{j}}{h}\right)_{e}=0.28(F r)^{1.75}(1+\theta)^{0.22}\left(\frac{t_{r}+t_{s}}{h}\right)^{0.44}\left[(1+\tan \alpha) \frac{A_{a}}{A_{w}}\right]^{-0.087}$

Where, $F r$ is the Froude number, $\theta$ is the ramp angle, $t_{r}$ is the ramp height (m), and $t_{s}$ is the step height $(\mathrm{m})$. They also presented the following expression to define scale effects between prototype and calculated values by Eq. 13 based on their laboratory tests

$\left(\beta_{c 1}\right)_{p}=\xi \beta_{m}^{\phi}$

Where, $\xi$ and $\phi$ are experimental constants, and the subscripts $p$ and $m$ indicate prototype and model values respectively.

The second values were calculated by Eq. 16 proposed by some other researches depending on the relative jet length [10]

$\beta_{c 2}=q_{a} / q_{w}=K\left(L_{j} / h\right)$

where $K$ is a dimensionless coefficient that is a function of the aerator geometry and dimensionless flow characteristics (i.e. Froude and Euler numbers)

Figure 4(a) show that the significant differences appear between the results of the CFD and the physical model because of the scale effects disregarded in experiments. When the unit discharges of air, $q_{a}$, are considered, the average rate of 1.30 is obtained between values of the CFD and the physical model. Escher and Siegenthaler [11] gave the rate of unit air discharges as a range of 1.11- 1.43 between the physical model and prototype. In Demiroz's [12] another experiment, this coefficient was taken as 1.40 when the scale of 1:30 was used. It is naturally expected that the scale effects of the model with 1:25 scale are less than the model with 1:30 scale. Therefore it is noted that the results of CFD model agrees with the prototype air entrainments represented by Demiroz's [2] model. The relationship of $\beta_{f} \approx 1.17 \beta_{m}$, in this study, was also derived with a correlation coefficient of 0.99 . In Figure $4(\mathrm{a}), \beta_{m}$ is the model values presented by Demiroz [2], and the value of $\beta_{f}$ is obtained from the CFD model using Fluent.

When the values of $\beta_{f}$ are compared with the values of $\beta_{c l}$ calculated by Eq. 13, the significant differences between both values appear as shown in Figure 4(b) because of scale effects. The constants in Eq. 15 were obtained as $\xi=5.221$ and $\phi=1.211$ with a correlation coefficient of 0.98 . These constants were determined by Kokpinar and Gogus [9] as $\xi=5.194, \phi=1.150$ for symmetrical aerators and $\xi=4.186, \phi=1.188$ for asymmetrical aerators.

$$
\left(\beta_{c 1}\right)_{p}=5.221\left(\beta_{c 1}\right)^{1.211}
$$

In order to calculate $\beta_{c 2}$ by Eq. 16, the jet lengths obtained from CFD analysis were used. The values of $\beta_{c 2}$ calculated by Eq. 16 with the $K=0.023$ are reasonably in good agreement with the CFD values of $\beta_{f}$. The values of $K$ in Eq. 16 are obtained by many researchers, for example, Hamilton [13] notes that the value of $K$ usually falls in the range of $0.01 \leq K \leq 0.05$. Coleman et al. [14] used the $K$ value of 0.02 in their design of aerators for the Uribante Dam spillway. For three aerators at Foz do Areia Dam spillway, the $K$ value was found to be 0.033 for symmetrical aeration condition, while the $K$ value of 0.023 was determined for an asymmetrical aeration condition [15]. Wei and De Fazio [16] determined the value of $K$ for Guri Dam spillway as varying from 
0.01 to 0.035 . Pinto and Neidert [10] found the $K$ values in the range of $0.01 \leq K \leq 0.08$. The coefficient of 0.023 determined in this study is too close the above values, and in agreement.

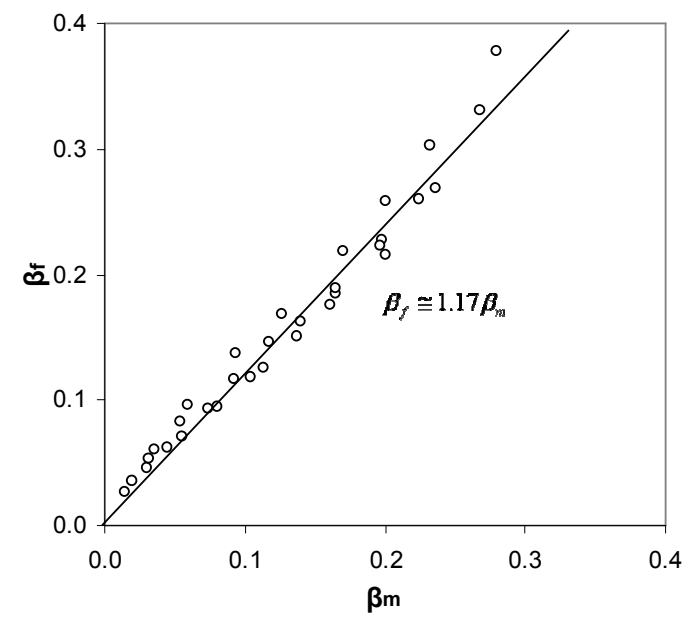

(a)

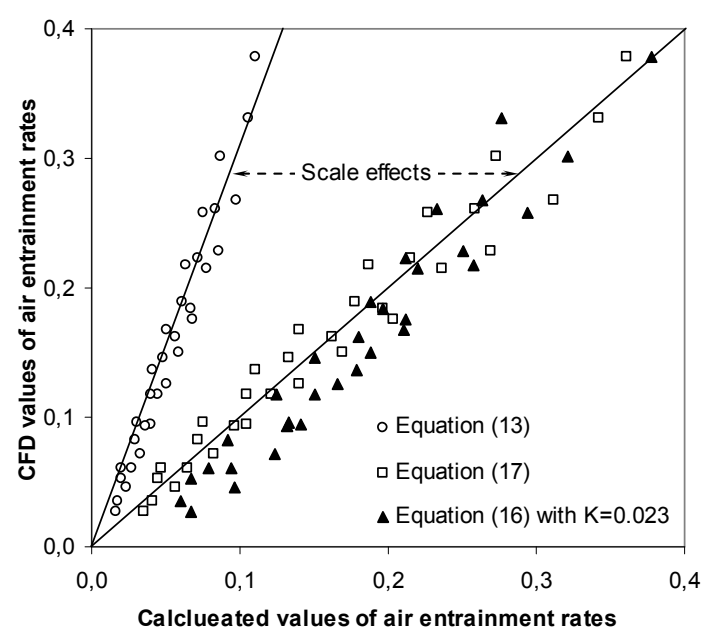

(b)

Fig. 4 (a) Comparison of the CFD values with experimental data

(b) Comparison of the CFD values with calculated data

\section{CONCLUSIONS}

The below conclusions can be given depending on the comparison and verification concerned with CFD model of the spillway aerator:

1. The results of CFD analysis by using Fluent are not in good agreement with the data of physical model including considerable scale effects. Nevertheless, the scale effect factor is considered in the laboratory data, it is clear that the both values will be in agreement.

2. The CFD results are reasonably in agreement with the results of empirical relationships presented by other researchers.

3. Therefore, it is proposed that the CFD models can be used for estimating of air entrainment in spillway aerators, even if it is required, together with physical models.

4. It should be said that the advanced numerical methods have got importance against physical models with recently developing computational methods. In this manner, the use of CFD models in hydraulic applications is also encouraged.

\section{LIST OF SYMBOLS}

$A_{a}:$ control area of air duct

$A_{w}:$ water flow area

$B$ : width of spillway chute

$E$ : Richardson error estimator

$e:$ approximate relative error

$F$ : body force $f_{1}$ : fine-grid solution

$f_{2}$ : coarse-grid solution

$\mathrm{Fr}$ : Froude number

$F_{S}$ : safety factor

$h$ : approaching flow depth

$L_{j}$ : jet length 
$n:$ number of phases

$N$ : number of cell.

$p:$ order of accuracy

$q_{a}:$ unit air discharge

$q_{w}:$ unit water discharge

$r:$ refinement factor

$t_{r}:$ ramp height

$t_{s}:$ step height

$\alpha$ : angle of chute slope to horizontal

$\beta$ : air entrainment rate $\theta:$ angle of aerator ramp

$\mu_{m}$ : viscosity of mixture

$\xi, \phi, K$ : experimental constants

$\rho_{m}:$ mixture density

$\vec{u}_{m}:$ mass-averaged velocity

$\bar{c}:$ a coefficient

$\vec{u}_{D k}:$ drift velocity

$\alpha_{k}$ : volume fraction of phase $k$

$\Delta V_{i}$ : volume of $i^{\text {th }}$ cell

\section{REFERENCES}

1. N. L. de S. Pinto, Model evaluation of aerators in shooting flow. Symposium on Scale Effects in Modelling Hydraulic Structure,. International Association for Hydraulic Researc,. Esslingen, Germany, 3-6 September, 4.2-1 to 4.2-6, 1984.

2. E. Demiroz, Determination of project criterions relating to adding aerators to structures for the aeration of spillway chute (in Turkish). State Water Works (DSI), Research and Development Chairmanship. Publication no: HI-754, Model no: M-212, Ankara, Turkey, 1986.

3. B. E. Launder and D. B. Spalding, Lectures in Mathematical Models of Turbulence. Academic Press, London, England, 1972.

4. FLUENT 6.2 USER'S GUIDE, FLUENT Inc., Lebanon, NH, 2005.

5. C. J. Freitas, Journal of Fluids Engineering Editorial Policy Statement on the Control of Numerical Accuracy, Journal of Fluids Engineering-Transactions of the ASME, 115 (3), 339-340, 1993.

6. P. J. Roache, Perspective a method for uniform reporting of grid refinement studies, Journal of Fluid Engineering 116, 405-413, 1994.

7. P. J. Roache, Quantification of the uncertainty in computational fluid dynamics, Annual Review Fluid Mechanics 29, 123-160, 1997.

8. P. J. Roache, Verification of codes and calculations, AIAA 36, 696-702, 1998.

9. M. A. Kokpinar and M. Gogus, High-speed jet flows over spillway aerators, Canadian Journal of Civil Engineering 29(6), 885-898, 2002.

10. N. L. de S. Pinto and S. H. Neidert, Evaluating entrained air flow through aerators, Water Power and Dam Construction 35(8), 40-42, 1983.

11. L. Eccher and A. Siegenthaler, Spillway aeration of aeration of the San Roque project, Water Power and Dam Construction 34(9), 37-41, 1982.

12. E. Demiroz, The model study report of the chute aeration of Ilisu Dam spillway (in Turkish). State Water Works (DSI), Research and Development Chairmanship, Publication no: HI-719, Model no: M-194, Ankara, Turkey, 1982.

13. W. S. Hamilton, Preventing cavitation damage to hydraulic structures: Part 3, Water Power and Dam Construction 36(1):42-45, 1984.

14. H. W. Coleman, A. R. Simpson and L. M. De Garcia, Aeration for cavitation protection of Uribante Spillway, Proceedings of the Conference on Frontiers in 
Hydraulic Engineering, American Society of Civil Engineers, Cambridge, MA, 9-12 August, 438-443, 1983.

15. N. L. de S. Pinto, S. H. Neidert and J. J. Ota, Aeration at high velocity flows, Water Power and Dam Construction 34(2), 34-38 and 34(3), 42-44, 1982.

16. C. Wei and F. DeFazio, Simulation of free jet trajectories for the design of aeration devices on hydraulic structures. Proceedings of $4^{\text {th }}$ International Conference on Finite Elements in Water Resources, IAHR, ISCME, DFG, Hanover, Germany, 1982.

17. M. C. Aydin, CFD analysis of bottom-inlet spillway aerators. Ph.D. Thesis, Department of Civil Engineering, Firat University, 2005. 